\title{
Preference ranking of screen layout principles
}

\author{
Carolyn Salimun ${ }^{1}$ \\ 1Department of Computing Science, \\ University of Glasgow, \\ Glasgow G12 8QQ, United Kingdom \\ \{carolyn\}\{hcp\}\{stephen\}@dcs.gla.ac.uk
}

\author{
David R. Simmons ${ }^{3} \quad$ Stephen Brewster ${ }^{4}$ \\ 2Department of Psychology \\ Aalto University and University of Helsinki \\ Glasgow G12 8QQ, United Kingdom \\ d.r.simmons@psy.gla.ac.uk
}

\begin{abstract}
This paper presents the results of a study on the preference ranking of six layout principles (Cohesion, Economy, Regularity, Sequence, Symmetry, and Unity). Preference judgments were conducted using a forced-choice paired comparisons method. The findings of the present study indicate that, contrary to suggestions in previous literature, an interface was most preferred when it found that the layout principles of Symmetry and Cohesion were more influential than the other layout principles. Further research is needed to identify other aesthetics factors which might influence preferences, and establish appropriate design guidelines
\end{abstract}

Aesthetics, Aesthetics measures, Preference, Screen design.

\section{INTRODUCTION}

The relationship between user preference and the aesthetics of interfaces has been widely studied in the literature e.g. [2, 5, 7-11, 13, 16], with findings that generally confirm that interfaces with highly rated look at) are preferred over relatively unaesthetic interfaces and that these same highly aesthetic interfaces are perceived as easier to use. The problem with these studies is that they do not specifically identify the aesthetic principles that affect user preference and the extent of their effect. In this paper, we are concerned with aesthetic principles for the layout of objects on a screen we are not concerned here with other visual principles that might affect the aesthetic perception of an interface such as color and font. Most of the previous studies investigating the relationship between user preferences and the aesthetics of an interface have been performed using website interfaces $[2,9,12,16]$, with some using MP3 skins [5], and multimedia application packages [10, 17]. With a few exceptions, though, these studies have not been specific on which of the aesthetic factors of an interface affect preferences. They do not indicate which aesthetic principles are more important than others, for example, that the Gestalt principle of grouping has been found to be more important than the Gestalt principle of symmetry. Some of these studies, for example de-Angeli and Sutcliffe [2], rely heavily on the aesthetic dimensions proposed by Lavie and Tractinsky[8] (classical aesthetics and expressive aesthetics) as a baseline for defining the aesthetic of an interface and investigate preferences based on these dimensions. Their results, however, do not relate to any specific well-defined aesthetic layout principles. Other studies have been more specific when trying to investigate studying visual aesthetics in $\mathrm{HCl}$, Kurosu and Kashimura [7] identified that user preference of the aesthetics of an interface was related to the perceptual grouping of Gestalt theory. They found that interfaces which followed the perceptual grouping principle were not only perceived as more aesthetic but also more usable. Their findings were similar to those of Ngo et. al [10] and Bauerly and Liu [1]. Ngo et. al and Bauerly and Liu proposed mathematical formulae based on Gestalt principles to assess the layout aesthetics of an interface. These mathematical formulae have been proven to be robust in measuring interface aesthetics (i.e. the computed aesthetic value for the interface correlates with the aesthetic ratings of human viewers [10]). These studies are very specific in identifying the aesthetic factors that influence preference; the 14 formulae are clearly defined and described. For example, there are aesthetic formulae for balance, symmetry and unity, all of which are based on the Gestalt theories. However, these studies do not prioritize these aesthetics principles according to their importance in preference judgments. Parizotto- Rebeiro and Hammond [13] conducted a preference study which investigated several Gestalt principles e.g. unity, homogeneity, proportion, balance, and rhythm. His results compared the effect of the extent of aesthetic presence within each layout principle (i.e. good unity vs bad unity), but did not compare the effect between principles (i.e. good unity vs good balance). The research reported here investigated the relationship between user preferences of the aesthetic design of interfaces, based on six of the aesthetics formulae defined by Ngo et. al - Cohesion, Economy, 
Regularity, Sequence, Symmetry, and Unity, with the intention of producing a ranked list of importance. The wide nature of our participants gave us an opportunity to further investigate the effect of culture, which has not been done before apart from the work of Tractinsky [18].

\section{METHODOLOGY}

The method of paired comparisons was used to obtain the preference of the participants for 15 different layouts. The aesthetic value for each of the 15 layouts used in this experiment was as shown in table 1. These 15 layouts are derived from the mathematical formulae developed by Ngo et. al. The explanations of these terms and the mathematical formulae are given in table 2 .

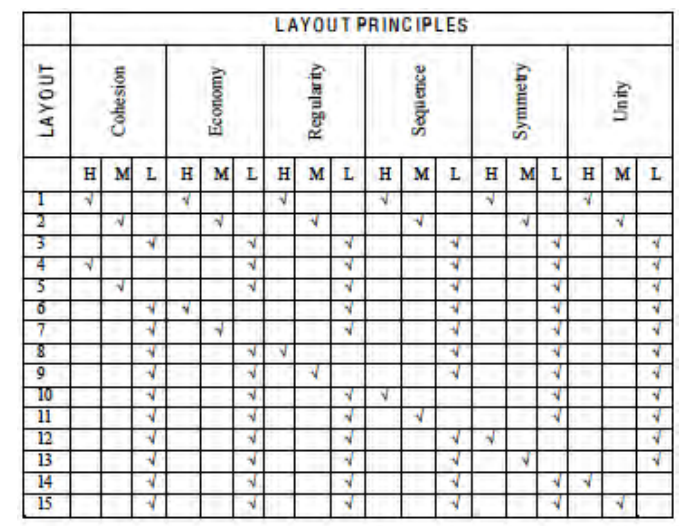

$\mathrm{H}=$ High, $\mathrm{M}=$ Medium, L=Low

$T$ able 1. The aesthetics value of each layout

$T$ able 2. Six formulae that were used to measure interface layout aesthetics ( $F$ or full details of how to calculate these parameters and for definitions of terms see Ngo et al [10])

$\mathrm{CM}=\frac{|\mathrm{CMI}|+|\mathrm{CMlo}|}{2} \in[0,1]$
$\begin{aligned} & \text { Cohesion is achieved by using a similar aspect ratio for the } \\ & \text { bounding box surrounding the interface objects and the } \\ & \text { frame in which it is viewed. }\end{aligned}$
$\mathrm{ECM}=\frac{1}{\text { nSize }} \in[0,1]$
$\begin{aligned} & \text { Economy is achieved by using as few sizes of the interface } \\ & \text { objects as possible }\end{aligned}$
$\begin{aligned} & \mathrm{RM}=\frac{|\mathrm{RMalignment}|+|\mathrm{RMspacing}|}{2} \in[0,1] \\ & \text { Regularity is achieved by establishing standard and } \\ & \text { consistently spaced horizontal and vertical alignment points } \\ & \text { for interface objects, and minimizing the alignment points. }\end{aligned}$

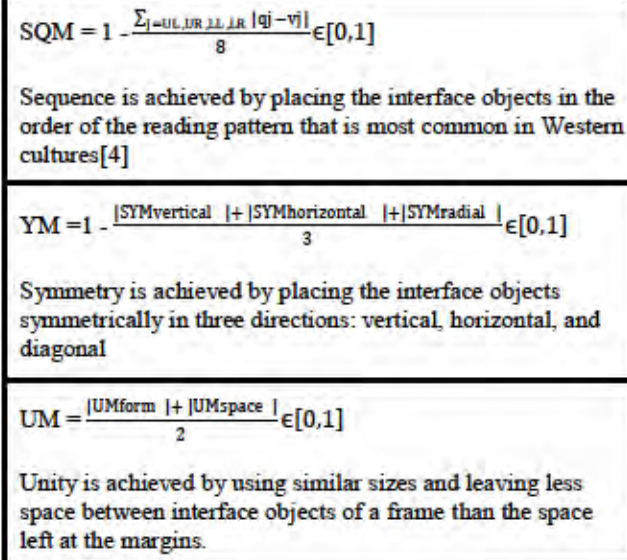

\subsection{Participants}

Atotal of 72 participants participated in this experiment in which 26 participants classified themselves as Asian, 42 as Western, and 4 as others. From the total of 72 participants, data from 15 participants ( 5 Asian, 10 Western) were discarded due to the high number of circular triads in their data (see below for an explanation). All the participants were computer literate and used computers daily. The participants received no remuneration for their participation.

\subsection{Task}

The participants were presented with a series of 105 pairs of pictures. For each pair they were required to report which of the two they preferred more.

\subsection{Variables}

There are three variables of interest in this experiment. The independent variables: aesthetics level (low, medium, high), aesthetics parameter (Cohesion, Economy, Regularity, Sequence, Symmetry, and Unity), and dependent variable: preferences score.

\subsection{Stimuli}

The program used to generate stimuli for the experiment was written in Java. The program randomly generated 15 different layouts of mixed inverted and upright triangles and calculated the aesthetics value. The triangles were drawn using black lines on a white background (see figure 1) and were $5-102 \mathrm{~mm}$ in height and $5-125 \mathrm{~mm}$ in width. Since the main focus of this experiment was on the layout aesthetics, the colors were limited to black (color of the triangle line) and white (background) to avoid or minimize the effects of confounding factors. Table 3 shows the aesthetic levels and the range of values of each aesthetic level. These values ranged between 0 (the worst) and 1 (the best). The aesthetic levels (low, medium, high) and the value range of each aesthetic level were as suggested by $\mathrm{Ngo}$ et al. The Java program calculated specific locations 
for each triangle such that the overall layout fulfilled the required layout principle (e.g. High Cohesion). Table 1 shows the aesthetic value of each of the 15 layouts. Figure 1, 2, 3, and 4 show examples of layouts with different aesthetic values.

In the previous studies by $\mathrm{Ngo}$ et. al [10], and Bauerly and Liu [1] differ between a model screen and a real screen. Consequently, in this experiment, it was assumed that the use of triangles to test the perceptions of more realistic layouts.

Table 3. Aesthetics value range for each level of aesthetics

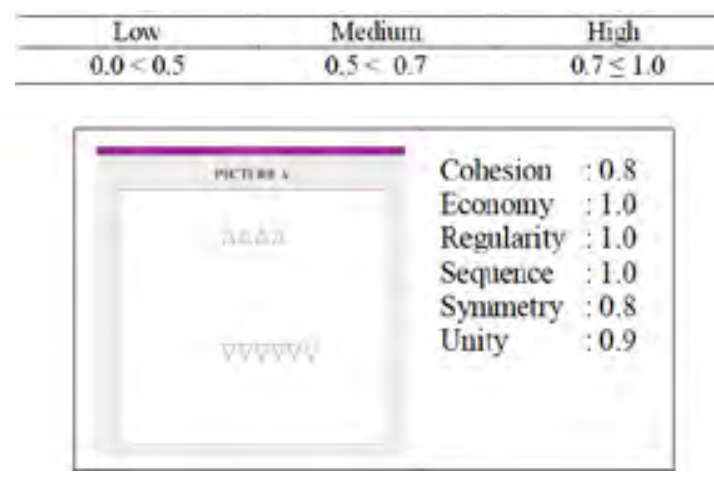

Figure 1. An interface layout with high aesthetics value in all parameters

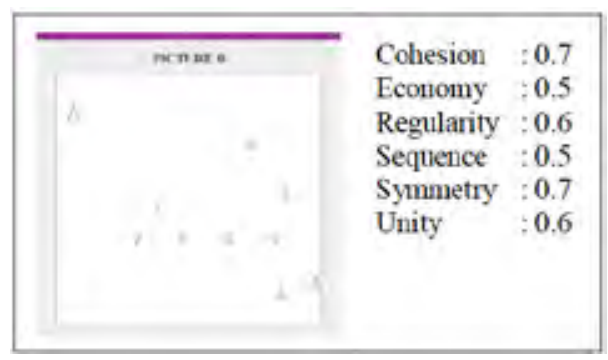

Figure 2. A n interface layout with medium aesthetics value in all parameters

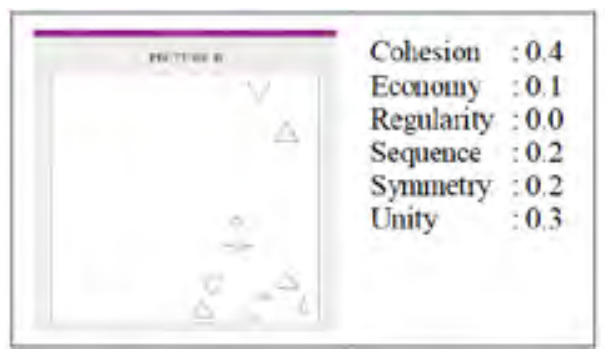

Figure 3. An interface layout with low aesthetics value in all parameters



Figure 4. An interface layout with high aesthetics in Regularity and low aesthetics in the remaining 5 other parameters

\subsection{Procedure}

At the beginning of the experimental session, the participants received written and verbal instructions, signed a consent form, and filled in a demographic questionnaire. Participants were then seated in front of a laptop (Screen size of $30 \mathrm{~cm}$ with resolution of $1024 \times 768$ pixels, viewed from approximately 50 $\mathrm{cm})$. A computer program, written in JAVA was used to present the stimuli and present the paricipants' choice of pictures (see Figure 5).

The experimenter started the program and demonstrated to the participant how to do the task. The purpose of the demo was to ensure that the participant was familiar and comfortable with the task before starting the experiment proper. After finishing the demo, the experimenter restarted the program and asked the participant to click on the start button whenever they were ready.

As the participant clicked on the start button, they were presented with a pair of pictures, labeled as picture $A$ and picture $B$. Picture A was displayed first followed by picture $B$, one at a time. Each picture was displayed for two seconds each before the participants made their choice of which of the two pictures they preferred the most by clicking on the appropriate button. The screen where the participants were required to make a choice was untimed; however they were not allowed backtrack. The next pair was shown automatically after the participants clicked on the answer button. This process continued until all 105 pairs of pictures were shown (15 stimuli each shown 14 times with each of the other stimuli).

The order of the pairs and the orders of the picture in each pair were both randomized to minimize learning effects.



Figure 5. The JAVA program

\section{RESULT}

The data from the experiment were analyzed using the program TRICIR (Circular Triad Analysis) [14]. This software analyzes circular triads (the inconsistency of choices e.g. $A>B, B>C, C>A$ ) in paired comparisons data and provides information on circular triads probabilities for individual judges and objects as well as judge and objects groups, performs object scaling according to the simplified rank method, and calculates Kendall's coefficients of consistence and concordance. 


\subsection{Coefficient of Consistency}

The coefficient of consistency is the consistency of one participant in making their choices, as measured by the absence of circular triads. Circular triads occur whenever inconsistent pair wise choices occur. For example, if three objects were compared $A, B$, and $C$, which produced three possible pairs, if $A$ is chosen over $(>) B$, and $B>C$, the choice of the third pair, should be $A>C$ instead of $C>A$. Circular triad occurs when $C>A$.

This is a useful value to calculate because a low value of the coefficient of consistency shows that the participant was either responding carelessly or was not competent in the task. A high value of the coefficient of consistency shows that the participant can make choices and that their view of the layouts is sufficiently different to enable a reasonably consistent set of preferences to be made, thus it can be confidently claimed that the preferences were not allotted at random.

The coefficient of consistency (w) was computed for each participant. Based on these values, data from 15 of the 72 participants were discarded as the coefficient of consistency was less than $0.50 \pm$ this cutoff was based on that used in previous studies [6]. The remaining 57 participants were highly consistent with the mean coefficients of consistency of 0.7016 and standard deviation of 15.107. The number of circular triads within these 57 participants ranged from 9 to 69 .

\subsection{Result for All Participants}

\subsubsection{Coefficient of agreements}

The coefficient of agreement refers to the measure of agreement in the object rankings between the judges. The value indicates the extent of consistency among the whole group of the participants. The coefficient of agreement is Kendall's Coefficient of Concordance (W) for Judges' Votes. For all 15 parameters this coefficient was very low $W=.1023$ (of possible 1.0). This means that there was a great deal of variation in the preference data between the participants.

\subsubsection{Ranking results for all participants}

Figure 6 shows the preference ranking of the 15 layouts made by the 57 participants, with 8 layouts being voted for less often than expected by chance and 7 layouts more often. The maximum votes possible for each layout was 798 which means if the number of votes was close to $399(50 \%)$ (see Figure 6) this might have arisen by chance. The numbers of votes for each layout are shown in table 5. Applying a chi-square test to this table yielded a chi square statistic of 162.91 which is greater than the critical value $(p=0.05)$ for $d f=14(x 2=23.685)$. Thus, it can be concluded that there is a significant difference between the data sets which is unlikely to be due to chance alone.

Further analysis shows that of the 15 layouts only 8 layouts were voted for significantly more or less often than another with a $p$ - value of 0.05 . These were All High, All Medium, Medium Sequence, Medium Regularity, High Sequence, High Cohesion, Medium Cohesion, Medium Symmetry (see Figure 6). Table 6 shows these pairs of layouts. It was surprising to find that the layout All High (computed value of aesthetics level was set as high in all of the six layout principles -refer to table 1) was voted as the least preferred compared to all other layout principles. The level of aesthetics. Table 6 also shows that there were higher preferences for layouts organized according to the principles of Cohesion and Symmetry as compared to other layout principles.

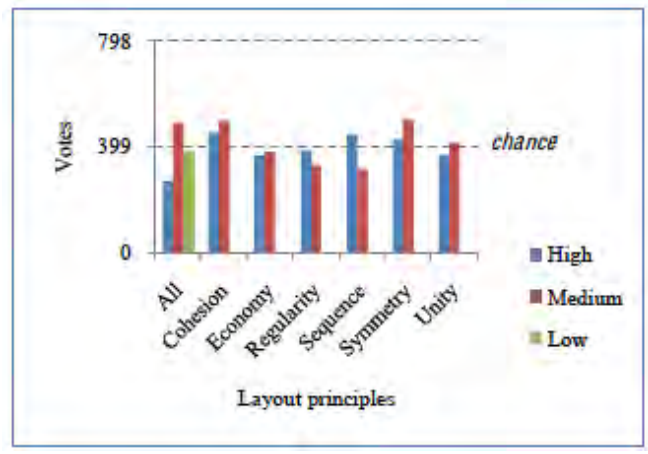

Figure 6. Preference ranking for the 15 layouts

Table 4. Votes for each layout for all participants

\begin{tabular}{lcc}
\hline Layouts & Number of votes & $\%$ \\
\hline All High & 268 & 34 \\
All Medium & 487 & 61 \\
All Low & 376 & 47 \\
High Cohesion & 450 & 56 \\
Medium Cohesion & 494 & 62 \\
High Economy & 364 & 46 \\
Medium Economy & 378 & 47 \\
High Regularity & 383 & 48 \\
Medium Regularity & 330 & 41 \\
High Sequence & 443 & 56 \\
Medium Sequence & 313 & 39 \\
High Symmetry & 424 & 53 \\
Medium Symmetry & 499 & 63 \\
High Unity & 365 & 46 \\
Medium Unity & 411 & 52 \\
\hline
\end{tabular}

Table 5. Pairs which differ significantly at $p<0.05$ for all participants

\begin{tabular}{lll}
\hline Layouts & & Layouts \\
\hline All Medium & is preferred to & All High \\
High Cohesion & is preferred to & All High \\
Medium Cohesion & is preferred to & All High \\
High Sequence & is preferred to & All High \\
Medium Symmetry & is preferred to & All High \\
All Medium & is preferred to & Medium Sequence \\
Medium Cohesion & is preferred to & Medium Regularity \\
Medium Cohesion & is preferred to & Medium Sequence \\
Medium Symmetry & is preferred to & Medium Regularity \\
Medium Symmetry & is preferred to & Medium Sequence \\
\hline
\end{tabular}




\subsection{Result for Asian Participants}

The Kendall's Coefficient of Concordance (W) for the votes of the 21 Asian participants was also low $\mathrm{W}=.1859$. Figure 7 shows the preference ranking of the 15 layouts, with 7 layouts voted for less often than expected by chance and 8 layouts voted for more often. The maximum votes possible for each layout was 294 which means if the number of votes was close to $147(50 \%)$ (See Figure 7) this might have arisen by chance. The numbers of votes for each layout are shown in table 7 . The Chi Square is 109.619 with $\mathrm{df}=14$ which is greater than the critical value $(p=0.05)$ of $x 2(23.685)$, indicating that the preference ranking as a whole is significantly different from chance.

Further analysis shows that of the 15 layouts only 4 layouts were voted for significantly more or less often than another with $p<0.05$. These were AllHigh, All Medium, Medium Cohesion, Medium Symmetry. Table 8 shows these pairs of layouts. Again, it was surprising to find that the layout All High (computed value of aesthetics level was set as high in all of the six layout principles refer to Figure 7) was voted as the least preferred compared to all other layouts. The other surprising finding was that the layouts was that the layouts with a 'medium' level of aethetics were more preferred than those with a 'high' level of aesthetics. hetics. Table 8 also shows that there were higher preferences for layouts organized according to the principles of Cohesion and Symmetry as compared to other layout principles.

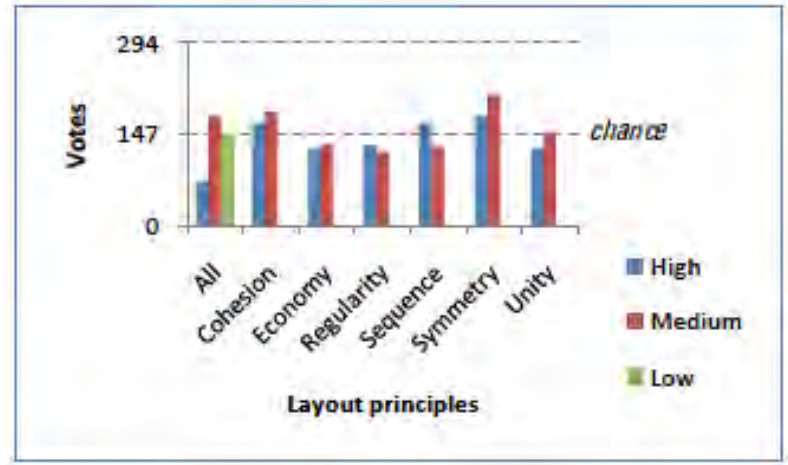

Figure 7 Asians' preference for the 15 layouts
Table 6. Votes for each layout for Asian participants

\begin{tabular}{lcc}
\hline Layouts & Number of votes & $\%$ \\
\hline All High & 72 & 23 \\
All Medium & 176 & 56 \\
All Low & 148 & 47 \\
High Cohesion & 166 & 53 \\
Medium Cohesion & 183 & 58 \\
High Economy & 123 & 38 \\
Medium Economy & 131 & 42 \\
High Regularity & 130 & 41 \\
Medium Regularity & 119 & 38 \\
High Sequence & 165 & 52 \\
Medium Sequence & 129 & 41 \\
High Symmetry & 176 & 56 \\
Medium Symmetry & 211 & 67 \\
High Unity & 125 & 40 \\
Medium Unity & 151 & 48 \\
\hline
\end{tabular}

Table 7. Pairs which differ significantly at $p<0.05$ for Asian participants

\begin{tabular}{lll}
\hline Layouts & Layouts \\
\hline All Medium (176) & is preferred to & All High (72) \\
Medium Cohesion (186) & is preferred to & All High (72) \\
High Symmetry (176) & is preferred to & All High (72) \\
Medium Symmetry (211) & is preferred to & All High (72) \\
\hline
\end{tabular}

\subsection{Result for Western Participants}

The Kendall's Coefficient of Concordance (W) for the votes of the 32 Western participants was also very low $\mathrm{W}$ $=.0843$. Figure 8 shows the preference ranking of the 15 layouts, with 8 layouts voted for less often than expected by chance and the remaining layouts voted for more often. The maximum votes possible for each layout was 448 which means if the number of votes was close to 224 $(50 \%)$ (see figure 8$)$ this might have arisen by chance. The numbers of votes for each layout are shown in table 9. The Chi Square is 75.98 with $\mathrm{df}=14$ which is greater than the critical value $\mathrm{x} 2$ (23.685), indicating that the preference ranking as a whole was significantly different from chance.

Further analysis shows that of the 15 layouts only 2 layouts were voted for significantly more or less often than another with $\mathrm{p}<0.05$ (table 10). These were All High, and Medium Cohesion in which All High was preferred less than Medium Cohesion.



Figure 8. Westerners' preferences for 15 layouts 
Table 8. Votes for each layout for Western participants

\begin{tabular}{lcc}
\hline Layouts & Number of votes & $\%$ \\
\hline All High & 157 & 35 \\
All Medium & 262 & 59 \\
All Low & 203 & 45 \\
High Cohesion & 257 & 57 \\
Medium Cohesion & 280 & 63 \\
High Economy & 213 & 48 \\
Medium Economy & 215 & 48 \\
High Regularity & 227 & 51 \\
Medium Regularity & 192 & 43 \\
High Sequence & 255 & 57 \\
Medium Sequence & 171 & 38 \\
High Symmetry & 224 & 50 \\
Medium Symmetry & 256 & 57 \\
High Unity & 210 & 47 \\
Medium Unity & 238 & 53 \\
\hline
\end{tabular}

Table 9. Pair which differ significantly at $p<0.05$ for Western participants

\begin{tabular}{|c|c|c|}
\hline 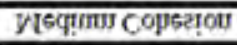 & 12 bLa|GLEq 10 & VII $\mathrm{H}$ ज़ा \\
\hline एवromf & & एकlonil \\
\hline
\end{tabular}

\section{ANALYSIS AND DISCUSSION}

The main objective of this research was to investigate the relationships between user preferences for the aesthetic design of interfaces, based on the six aesthetics formulae defined by Ngo et. al (Cohesion, Economy, Regularity, Sequence, Symmetry, and Unity), with the intention of producing a ranked list of importance. The forced-choice paired comparison method was used to obtain choices from the participants of which layout principles they preferred the most.

It was expected that layouts with high aesthetics levels would receive the most votes followed by the layout principles with medium aesthetics levels, and then the lower. This assumption was based on findings from previous literature which found that high aesthetic interfaces are more preferred than low aesthetic interfaces $[1,10]$. Our result however was, surprisingly, contrary to this expectation, as interfaces were more preferred when the layout of the interface was designed according to a medium level of aesthtics. This preference in which 'medium' aesthetics layouts were, on the whole, more preferred than high aesthetics is unlikely to be due to random fluctuations in the data, based on our analysis (table 5). One interpretation of these results is that interfaces which are highly 'orderly' or 'tidy' are less preffered than interfce which are slightly 'disordered' or 'messy'. We concluded that any extremely 'beautiful' or extremely 'ugly' interfaces are less preferred - thus extremes negatively affect the 'aesthetics experiance'.

Layouts that are completely 'disorderly' or too 'messy' might be perceived as too complicated, which leads to the feeling of discomfort or difficulty for the eyes in navigating through the interface. On the other hand, a layout which is too 'orderly' or 'tidy', although it promotes comfort and is easier for the eyes to navigate, makes the interface look too regular or ordinary and does not have a 'mysterious' effect which is important in arousing interest in the interface. Gaver, et al [3] suggest that ambiguity in an interface is not always bad. It can be intriguing, mysterious, and delightful and can encourage close personal engagement with the system. On the other hand, a layout which is too neat and regular does not arouse interest in the interface. Their study however does not mention to what extent the ambiguity of interface is perceived as mysterious and delightful, rather than discomforting: it is clear that a balance between intrigue and discomfort is needed.

Although the overall co-efficient of agreement among the participants was low $\mathrm{W}=.1023$, there was some consistency in the judgments which allows at least a partial ranking of the layout principles. The most preferred layout was Medium Symmetry (499 votes), followed by Medium Cohesion (494 votes). Medium Sequence and Medium Regularity were less preferred. But with the exception of the All High condition, the effects of variations in the layout conditions were relatively modest.

The variations in performance due to cultural background were also relatively modest. The Asian participants as a group were more consistent with each other, with a higher co-efficient of agreement than the Western participants, although this could be partially confounded by the smaller sample size. Whilst both groups demonstrated the lack of preference for the All High condition only the Asian participants showed any significant preferences for other layouts, with Medium Symmetry again being ranked as highest.

The high preference for Symmetry is not surprising. In fact it has been demonstrated not only in the study of interfaces [1] but also in human faces and animals (as cited in [15]). Reber et al [15] in their study investigating factors that influence aesthetics judgments suggested that the high preference of symmetry was influenced by the perceptual fluency (e.g. the ease of identifying the physical identity of the stimulus). Compared to other patterns, symmetrical patterns contain less information which makes them easier to process hence increasing the speed of processing fluency (Garner,1974 as cited by [15]). The higher the processing speed of perceptual fluency, the more positive the aesthetic judgments. What implications, then, do these results have for interface layout design? Clearly the use of triangle patterns is not exactly the same as using stimuli more likely to appear on a computer interface like image icons or blocks of text, so results might be affected should there be more content in the items that comprise the layout. However, in experimental terms this would also act as a confound, so the use of simple 'ininformative' visual objects for layout assessment seems justified. There is also the potential for idiosyncratic responses in this 
experiment since only one example layout was used for each condition. Nevertheless, it is clear from these results that 1 ) preferences were affected by the interface layout, and 2) the interface was more preferred when the layout was designed, in terms of Ngo et al's formulae, with a medium level of aesthetics. Should the purpose of the interface be to make the users enjoy the 'look' of the interface, the designer should emphasize Symmetry and Cohesion or design the interface with a medium level of aesthetics in all the tested layout principles. In more colloquial terms, the results suggest that designers should aim for something that is not obviously highly structured but that is also not messy (see figures 1-3).

Further research is needed, however, to identify other aesthetics factors that might influence preferences, to extend this area of research to more realistic interfaces, and establish appropriate design guidelines that will maintain the users' interest on the interface.

\section{REFERENCES}

[1] Bauerly, M., and Liu, Y. 2006. Computational modeling and experimental investigation of effects of compositional elements on interface and design aesthetics. International Journal of HumanComputerStudies 64,8, 670-682.

[2] De-Angeli, A., Sutcliffe, A., and Hartmann, J. Interaction, usability and aesthetics: what influences users' preferences?2006. In Proceedings ofthe 6th conference on Designing Interactive systems,ACM, 271-280.

[3] Gaver, W. W., Beaver, J., and Benford, S. Ambiguity as a resource for design.2003. In Proceedings ofthe SIGCHI conference on Human factors in computing systems,ACM, 233-240.

[4] Goldstein, E. B. 2007. Sensation and Perception Wadsworth: Belmont, CA.

[5] Hassenzahl, M. 2004. The Interplay of Beauty, Goodness, and Usability in Interactive Products. Human-Computer Interaction 19,4, 319 • 349.

[6] Krenz, R. D. 1964. Paired Comparisons as Applied to Seeding Cropland to Grass. Journal of Farm Economics 46,5, 1219.

[7] Kurosu, M., and Kashimura, K. Apparent usability vs. inherent usability: experimental analysis on the determinants of the apparent usability. 1995. In Conference companion on Human factors in computing systems, ACM, 292-293.

[8] Lavie, T., and Tractinsky, N. 2004. Assessing dimensions of perceived visual aesthetics of web sites. International Journal of Human-ComputerStudies 60,3, 269-298.

[9] Lindgaard, G., Fernandes, G., Dudek, C., and Browñ, J. 2006. Attention web designers: You have 50 milliseconds to make a good first impression! Behaviour \& Information Technology 25,2, 115-126. [10] Ngo, D. C. L., Teo, L. S., and Byrne, J. G. 2003. Modelling interface aesthetics. Information Sciences 152, 25-46.

[11] Norman, D. 2002. Emotion \& design: attractive things work better. Interactions 9,4, 36-42.

[12] Pandir, M., and Knight, J. 2006. Homepage aesthetics: The search for preference factors and the challenges of subjectivity. Interacting with Computers 18,6, 1351-1370.

[13] Parizotto-Rebeiro, R., and Hammond, N. 2005. Does Aesthetics Affect WKH 8VHUTV 3HUFHSWLRQV RI 9/(TV" Workshop on motivation and affect in educational software.

[14] Peter Dunn-Rankin, G. A. K., Susan R. Wallace, Shuqiang Zhang. 2004. Scaling Methods, Psychology Press.

[15] Reber, R., Winkielman, P., and Schwarz, N. 1998. Effects of perceptual fluency on affective judgments. American Psychological Society9,1, 4548.

[16] Schenkman, B. N., and Jonsson, F. U. 2000. Aesthetics and preferences of web pages. Behaviour \& Information Technology 19,5, 367-377.

[17] Szabo, M., and Kanuka, H. 1999. Effects of violating screen design principles of balance, unity, and focus on recall learning, study time, and completion rates. Journal of Educational Multimedia and Hypermedia 8,1, 23-42.

[18] Tractinsky, N. Aesthetics and apparent usability: empirically assessing cultural and methodological issues.1997. In The SIGCHI conference on Human factors in computing systems,ACM, 115-122. 